\title{
MODELING OF PHOTOTRANSDUCTION
}

\section{IN VISION SYSTEMS}

Lei Lu

Dr. Jinglu Tan, Thesis Supervisor

\begin{abstract}
To improve the usefulness of the electroretinogram (ERG) in identifying the sites and mechanisms of adaptation, development and disease processes, a quantitative model was developed based on the biochemical reaction kinetics in the phototransduction cascade and experimental data. A set of differential equations were derived to predict the electrical response of photoreceptors to light and system identification was employed to determine the model parameters. When applied to wild-type and retina-damaged mice, the proposed model effectively described the ERG a-wave over a range of light intensities and different stimulus patterns. From the model parameters, the retinadamaged subjects could be differentiated from the normal ones. It is anticipated that this model could help detect changes in the phototransduction process and enhance the utility of ERG in the clinic. It also provides insights into dynamics of the vision system.
\end{abstract}

